\title{
Evacuation time estimate for total pedestrian evacuation using a queuing network model and volunteered geographic information
}

\author{
Bharat Kunwar, Filippo Simini, and Anders Johansson \\ Faculty of Engineering, University of Bristol, Queen's Building, University Walk, Bristol, City of Bristol BS8 1TR, United Kingdom
}

(Received 22 September 2015; revised manuscript received 15 January 2016; published 10 March 2016)

\begin{abstract}
Estimating city evacuation time is a nontrivial problem due to the interaction between thousands of individual agents, giving rise to various collective phenomena, such as bottleneck formation, intermittent flow, and stop-andgo waves. We present a mean field approach to draw relationships between road network spatial attributes, the number of evacuees, and the resultant evacuation time estimate (ETE). Using volunteered geographic information, we divide 50 United Kingdom cities into a total of 704 catchment areas (CAs) which we define as an area where all agents share the same nearest exit node. $90 \%$ of the agents are within $\approx 6,847 \mathrm{~m}$ of CA exit nodes with $\approx 13,778$ agents/CA. We establish a characteristic flow rate from catchment area attributes (population, distance to exit node, and exit node width) and a mean flow rate in a free-flow regime by simulating total evacuations using an agent based "queuing network" model. We use these variables to determine a relationship between catchment area attributes and resultant ETEs. This relationship could enable emergency planners to make a rapid appraisal of evacuation strategies and help support decisions in the run up to a crisis.
\end{abstract}

DOI: 10.1103/PhysRevE.93.032311

\section{INTRODUCTION}

Interaction between individual agents, city topology, disaster type, evacuation mode, information propagation patterns, and stochastic variables can all influence the temporal extent of a city-wide evacuation. Additionally, growing urban populations [1] amplify the impact of extreme events [2]. There is a need to examine factors affecting evacuation time in relation to the latest understanding of crowd dynamics and evacuation behavior.

Evacuation time estimate (ETE) analysis (a) tells emergency planners if an evacuation plan can reduce hazard exposure time, (b) measures the effect of uncontrollable events such as adverse weather, and (c) assesses whether traffic management actions help reduce it [3]. A study of flood evacuation in the Netherlands identifies a need for alternative evacuation strategies for coastal areas after it found that it was not feasible to evacuate preventively within a $48 \mathrm{hr}$ warning window [4]. EMBLEM2, an empirical study, categorizes research findings about evacuees' behavior in hurricanes into 4 evacuation route system parameters, 16 behavioral parameters, and 5 evacuation scope and/or timing parameters to calculate ETEs [5]. A sensitivity analysis of radiological emergency microtraffic simulation finds that the ETE is sensitive to traffic factors (interaction with pedestrians, intersection traversing time, car ownership, etc.) and route choice mechanisms (shortest path and myopic behavior) [6]. NETVACl, a macrotraffic simulation, finds that ETEs for areas surrounding nuclear power plant sites are sensitive to road network topology, intersection design and control, and a wide

\footnotetext{
${ }^{*}$ Corresponding author: b.kunwar@bristol.ac.uk
}

Published by the American Physical Society under the terms of the Creative Commons Attribution 3.0 License. Further distribution of this work must maintain attribution to the author(s) and the published article's title, journal citation, and DOI. array of evacuation management strategies [7]. Another study produces ETEs for a 10 mile radius around 52 nuclear power stations taking consideration of factors such as population density, weather conditions, warning time, response time, and confirmation time [8].

Some models take a dynamic network flow approach to minimize evacuation time $[9,10]$ while others use social force based models like EPES to establish optimal earthquake evacuation behavior [11]. The "Last-Mile" project uses a "queuing network" model to obtain an optimal evacuation plan for the Indonesian city of Padang using time-dependent network attributes to imitate conditions of a tsunami [12]. The underlying flow model simulates traffic, taking only free speed, bottleneck capacities, and space constraints into account. This was adapted from an early queuing model for cars [13] that was applied to the city of Portland to assess traffic jams. This approach is preceded by an early evacuation plan optimization study for Yokosuda city in Japan which uses a combination of the shortest path algorithm and minimal cost flow approach accounting for the capacity limit of each place of refuge [14].

Evacuees' behavior plays an important role during evacuations. A combination of individual traits and basic social psychological processes such as (a) risk perception, (b) social influence, and (c) access to resources predict evacuation behavior while some population subgroups choose not to evacuate depending on the severity of the storm, territoriality, etc. according to a study conducted after Hurricanes Hugo and Andrew [15]. Subjective perception of how bad the storm is going to be and the severity of damage also seem to play an important role in evacuation likelihood following a warning [16]. The effect of compliance behavior on ETEs has been studied using the EVAQ evacuation model and a case study of the Rotterdam metropolitan area in the Netherlands [17].

Crowd dynamics is an important feature in large cities, and understanding it is a crucial component of emergency evacuation modeling where agent based modeling $(\mathrm{ABM})$ is increasingly being used for large scale simulations to account 
for many interacting entities [18]. The transition between low and high density phases is common in social systems like cities [19]. Keeping a constant lower limit on the net-time headway has been shown as one of the key mechanisms behind emergent crowd dynamics [20]. Observed collective phenomena in pedestrian crowds include lane formation in corridors and oscillations at bottlenecks in normal situations as well as different kinds of blocked states produced in panic situations [21]. Video recordings of the crowd disaster in Mina/Makkah during the Hajj on January 12, 2006, reveal two subsequent, sudden transitions from laminar to stop-andgo [22] and turbulent flows [23]. The transition to turbulent flow is responsible for sudden eruptions of pressure release comparable to earthquakes, which cause sudden displacements and the falling and trampling of people [24]. However, from a macroscopic viewpoint, pedestrian behavior can be assimilated into a relationship between walking speed $v$ and local density $k$, variables familiar to the transport research community [25].

Review of existing work highlights a gap in understanding which relates ETEs to the interaction between city population and their topological make-up. Topologies can vary among parts of cities, one city to another, and one region of the planet to another, all growing in complexity at the same time. A queuing network ABM which incorporates pedestrian behavior and network topology has the potential to define a direct relationship between city topological attributes and their ETEs.

\section{METHODOLOGY}

We will now describe a model used for deriving the necessary quantities required for our analysis. We make the following assumptions across the model:

(a) The evacuation type is a total evacuation scenario, for which exit nodes lie at intersections between major roads and the city administrative boundary [26].

(b) The evacuation mode is by walking only.

(c) The route to an exit node is calculated using Dijkstra's shortest path algorithm [27] (no dynamic routing to avoid congestion at bottlenecks).

We incorporate Weidmann's fundamental diagram to describe pedestrian behavior [25] shown in Fig. 1 into the model. Equation (1) describes the relationship between density $k$ and velocity $v$ in the range $0.0 \leqslant k \leqslant k_{v \text {, min }}$ ped $/ \mathrm{m}^{2}$ where the minimum velocity threshold for density $k_{v, \min }=5.0 \mathrm{ped} / \mathrm{m}^{2}$. When $k=0.0 \mathrm{ped} / \mathrm{m}^{2}$, the free-flow velocity $v_{f}$ is $1.34 \mathrm{~m} / \mathrm{s}$. However, when $k>k_{v \text {, min }}, v_{\text {min }}=v\left(k_{v, \min }\right)=0.04 \mathrm{~m} / \mathrm{s} \mathrm{such}$ that the minimum velocity is never less than $v_{\min }=0.04 \mathrm{~m} / \mathrm{s}$ (or $2.25 \mathrm{~m} / \mathrm{min}$ ) which we draw from a limited set of empirical observations of high density crowds [20] which is still not very well understood. This has a physical implication that a minimum clearing must be available in front of an individual before he is able to make a decision to act. Without this kind of limit, individuals would be allowed to make infinitesimally small progress per time step which is neither plausible nor resolvable as simulations will tend to run indefinitely since $v \rightarrow 0 \mathrm{~m} / \mathrm{s}$ as we approach a maximum density of $k_{\max }=$ $5.4 \mathrm{ped} / \mathrm{m}^{2}$ if we evaluate $v\left(k_{\max }\right)$ using Eq. (1):

$$
v(k)=v_{f}\left(1.0-e^{-1.913\left(\frac{1.0}{k}-\frac{1.0}{k_{\max }}\right)}\right) .
$$
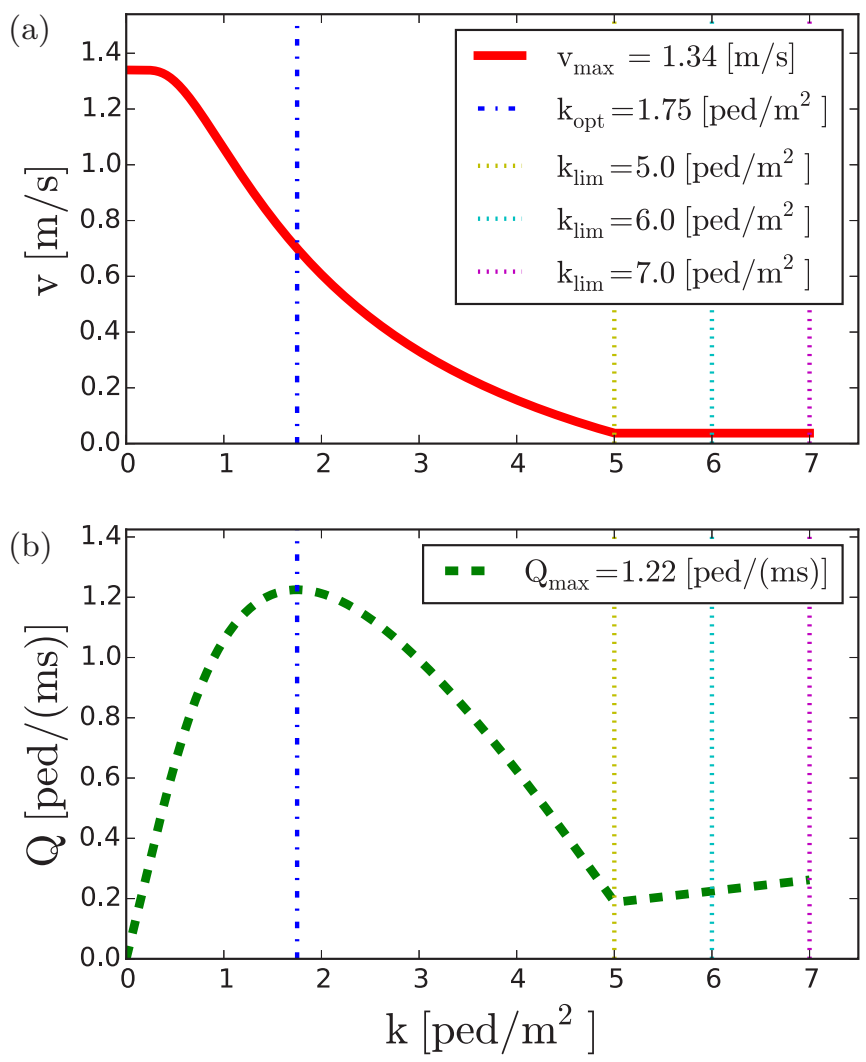

FIG. 1. Pedestrian fundamental diagram where (a) shows the relationship between density $k$ and velocity $v(k)=v_{f}(1.0-$ $\left.e^{-1.913\left(\frac{1.0}{k}-\frac{1.0}{k_{\max }}\right)}\right)$ [25] between $0.0 \leqslant k \leqslant k_{v, \min } \mathrm{ped} / \mathrm{m}^{2}$ where $k_{v, \text { min }}=5.0 \mathrm{ped} / \mathrm{m}^{2}$. In this equation, the free-flow velocity $v_{f}=$ $1.34 \mathrm{~m} / \mathrm{s}$. At maximum density $k_{\max }=5.4 \mathrm{ped} / \mathrm{m}^{2}, v=0 \mathrm{~m} / \mathrm{s}$. However, when $k>k_{v \text {, min }}, v_{\text {min }}=v\left(k_{v, \text { min }}\right)=0.04 \mathrm{~m} / \mathrm{s}$. On the other hand, (b) shows the relationship between density $k$ and flow $Q(k)=$ $k v(k)=k v_{f}\left(1.0-e^{-1.913\left(\frac{1.0}{k}-\frac{1.0}{k_{\max }}\right)}\right)$ [25]. From this equation, we can derive an optimum density $k_{\mathrm{opt}}=1.75 \mathrm{ped} / \mathrm{m}^{2}$ when the corresponding flow is a maximum of $Q_{\max }=1.22 \mathrm{ped} / \mathrm{ms}$. Both (a) and (b) also mark the position of $k_{\lim }$ at 5.0,6.0 and $7.0 \mathrm{ped} / \mathrm{m}^{2}$ for the purpose of sensitivity analysis.

The relationship between density $k$ and flow rate $Q$ follows as Eq. (2). We can differentiate this equation to derive the optimum density $k_{\mathrm{opt}}=1.75 \mathrm{ped} / \mathrm{m}^{2}$ when $d Q / d k=0$. The corresponding maximum flow rate $Q_{\max }=1.22 \mathrm{ped} / \mathrm{ms}$.

$$
Q(k)=k v(k)=k v_{f}\left(1.0-e^{-1.913\left(\frac{1.0}{k}-\frac{1.0}{k_{\max }}\right)}\right) .
$$

We use an adaptation of the queuing network model $[12,13,26]$ originally designed for vehicular traffic. The model follows the given rules for each remaining agent per time step until there are no more agents left:

(1) Calculate the link density using the area and occupancy of the link that the agent is on.

(2) Traverse the link at a velocity corresponding to the density using the fundamental diagram [25].

(3) If the agent reaches end of the link, go to the next link if there is space available.

(4) If there is no space available, remain in place.

(5) If the agent reaches the final destination, remove the agent from the simulation. 
The fundamental diagram is not able to describe system dynamics far from equilibrium (i.e., high density crowds) because it is an ensemble of a range of pedestrian behavior. Therefore, we model movement at high densities by implementing a network link density limit of $k_{\text {lim }}=5.0 \mathrm{ped} / \mathrm{m}^{2}$ as well as a minimum velocity threshold for the density at $k_{v, \min }=5.0 \mathrm{ped} / \mathrm{m}^{2}$. However, the simulation dynamics may be sensitive to different values of $k_{\mathrm{lim}}$. As such, we aim to compare the results obtained from a scenario with a density limit of $k_{\lim }=5.0 \mathrm{ped} / \mathrm{m}^{2}$ with alternative scenarios where $k_{\lim }=6.0$ and $7.0 \mathrm{ped} / \mathrm{m}^{2}$. If we maintain the minimum velocity threshold at $k_{v, \min }=5.0 \mathrm{ped} / \mathrm{m}^{2}$ as indicated in Fig. 1, this corresponds to a flow threshold of $0.20,0.24$, and $0.28 \mathrm{ped} / \mathrm{ms}$ at network link density limits of $k_{\lim }=5.0,6.0$, and $7.0 \mathrm{ped} / \mathrm{m}^{2}$, respectively.

Under these assumptions, we select 50 cities similar in area to the City of Bristol $\left(235.82 \pm 25 \% \mathrm{~km}^{2}\right)$. We use network topology approximated from OpenStreetMap (OSM) [28]. OSM is a source of volunteered geographic information (VGI) [29], growing in both contributor base and data quality [30-32]. We further divide these cities into 704 catchment areas (CAs), which we define as network components that emerge as agents are assigned to an exit node nearest to their initial position calculated using Dijkstra's shortest path algorithm [27]. Figure 2(a) illustrates CA formation for the City of Bristol and Fig. 2(b) shows how the distribution of the initial agent distance to their exit node $D$ varies between different CAs.

\section{A. Characteristic variables}

Characteristic variables independent of dynamic agent interaction informs part of our analysis. The first of these is the characteristic flow rate $Q_{c}$ described by Eq. (3). It is defined as free-flow time averaged flow whereby we assume infinite link capacity. As a consequence, $Q_{c}$ may sometimes exceed $Q_{\max }$. To illustrate the point, we mark the position of $Q_{c}$ for an example CA in Fig. 4(a).

$$
Q_{c}=\frac{v_{\mathrm{f}}}{D^{90 \%}} \frac{N}{W}=\frac{N}{T_{f}^{90 \%} W} .
$$

We calculate $Q_{c}$ using CA population $N$, exit node width $W$, and free-flow catchment area traversal time for $90 \%$ of all CA agents, $T_{f}^{90 \%}$, which is also our second characteristic variable. We estimate $N$ from GRUMPv1 year 2000 population data set [33] uniformly scaled up by a factor of $9.37 \%$ in order to account for the rise in United Kingdom (UK) population between the years 2000 and 2015 [34]. It has a granularity of $1 \mathrm{~km}^{2}$. Figure 3(a) shows how $N$ is distributed in log scale. Values range from $10^{2}$ to $10^{5}$, with a mean value of $10^{4.14} \approx 13,778$ agents. For exit nodes tagged "motorway" on OSM, we assume $W=7.5 \mathrm{~m}$ and for those tagged "trunk" or "primary", $W=5.0 \mathrm{~m}[26] . T_{f}^{90 \%}=D^{90 \%} / v_{f}$, where $D^{90 \%}$ is the distance to exit node for $90 \%$ of all agents and free-flow velocity $v_{f}=1.34 \mathrm{~m} / \mathrm{s}$. $T_{f}^{90 \%}$ is also marked in Fig. 4(a). If we ignore all congestion and bottleneck effects, it provides a lower bound estimate of evacuation time for $90 \%$ of all CA agents. We use $D^{90 \%}$ because it approximates the size of a CA as a scalar without the weight of the last decile skewing the result. Figure 3(b) shows how $D^{90 \%}$ is distributed across all

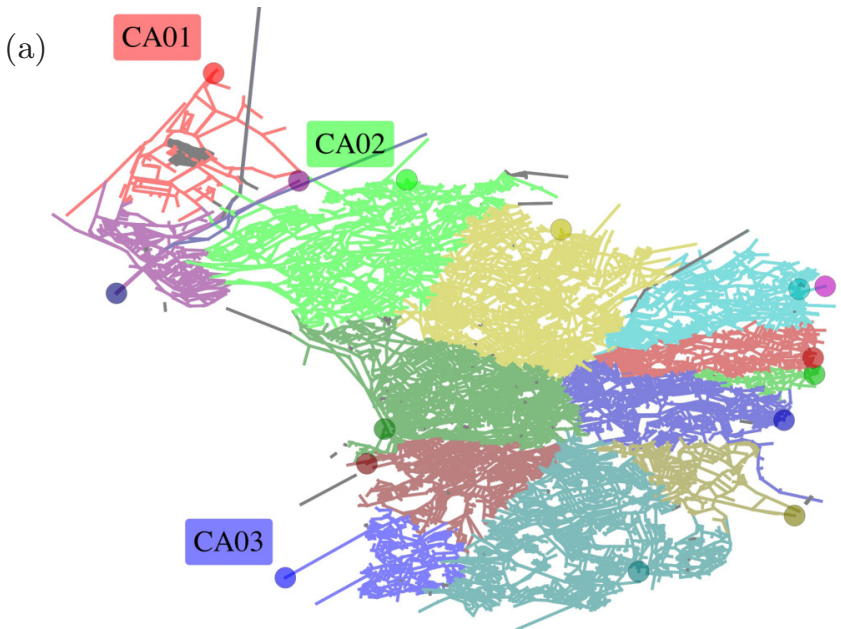

(b)

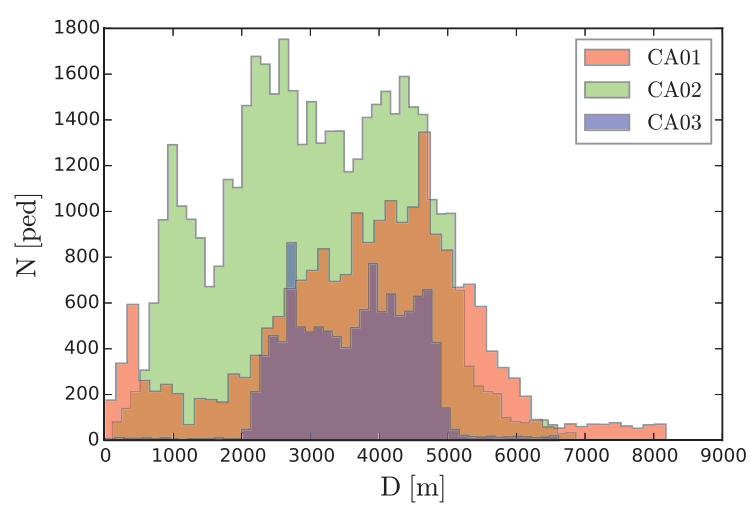

FIG. 2. (a) CAs are obtained by allocating agents to the exit node nearest to their initial position. For this, we use Dijkstra's shortest path algorithm [27]. Each color in the figure represents one of the 15 City of Bristol CAs. (b) Example showing distribution of agent distance to exit node $D$ for 3 City of Bristol CAs denoted by red (CA01), green (CA02), and blue (CA03) histograms.

CAs in log scale with values ranging between $10^{1.5}$ to $10^{4.5} \mathrm{~m}$ with a mean value of $10^{3.84} \approx 6,847 \mathrm{~m}$, a distance belt within which $90 \%$ of all CA agents are situated.

\section{B. Simulated variables}

Simulated variables are obtained by studying the dynamic interaction of agents under the following assumptions:

(i) All agents walk to the nearest exit on a signal to evacuate (i.e., premovement time is zero). (a)

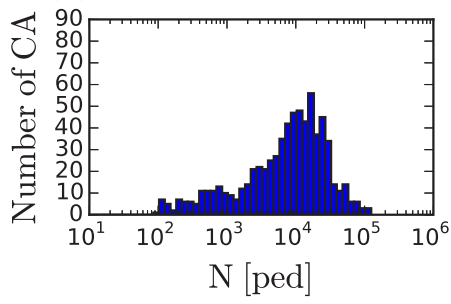

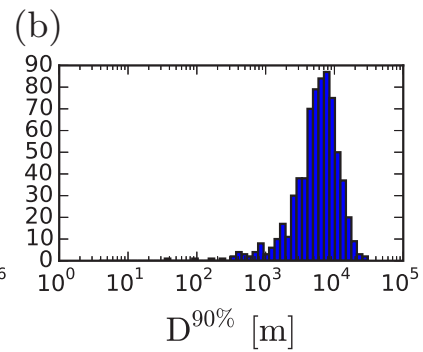

FIG. 3. (a) Histogram of CA populations $N$. (b) Histogram of agent distances to exits for $90 \%$ of all CA agents $D^{90 \%}$. 
(a)

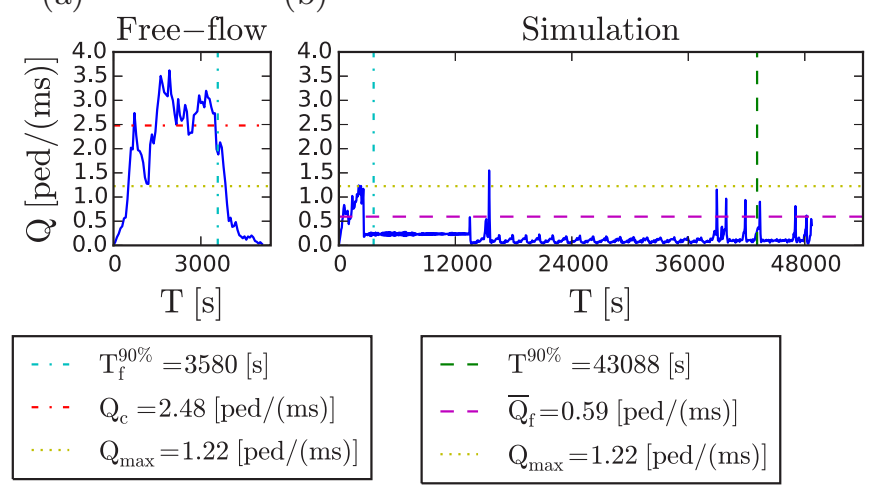

FIG. 4. An example using a City of Bristol CA, specifically CA02 from Fig. 2, to show the position of (a) the free-flow time for $90 \%$ of all CA agents, $T_{f}^{90 \%}$, the characteristic free-flow time averaged flow rate $Q_{c}$, (b) the simulated time for $90 \%$ of all CA agents, $T^{90 \%}$, and the mean of simulated exit node flow rate in the free-flow regime, $\bar{Q}_{f}$. Both (a) and (b) also specify the position of maximum flow rate $Q_{\max }$ which is exceeded in the free-flow case due to noninteraction between agents. However, the flow remains below this threshold during the simulation as link densities cannot be exceeded.

(ii) All agents act independently (complex social behaviors such as family regrouping, co-operation, etc. are not taken into account).

Our first simulated variable $\bar{Q}_{f}$ is defined as simulated exit node flow rate $Q$ averaged within the free-flow regime $\left(T<T_{f}^{90 \%}\right)$. The area under the flow curve for each CA is proportional to the total number of agents. The larger this area is before flow transitions to the congested phase, the bigger the $\bar{Q}_{f}$ value, precipitating a shorter congestion. Hence, the overall ETE is proportional to $\bar{Q}_{f}$. We show the position of $\bar{Q}_{f}$ for an example CA in Fig. 4(b). The flow curve $Q$ it is derived from is calculated using Eq. (2) where the density parameter $k=N /(W L), N$ is the number of agents arriving at the exit node per time step, $W$ is the width, and $L$ is the length of the exit link. Figure 4(b) also marks the position of $T^{90 \%}$, defined as the time at which $90 \%$ of all CA agents arrive at the exit node. Unlike $T_{f}^{90 \%}, T^{90 \%}$ takes agent interaction and emergent bottlenecks into account. While bottlenecks may be interspersed throughout a CA as shown by the example in Fig. 5 where observed local density $k$ varies through distance from exit node $D$ and elapsed time $T$, it is ultimately the exit node flow rate $Q$ that influences the overall ETE. Figure 5 also illustrates how velocity drops where the density is high for a randomly picked agent trajectory.

\section{RESULTS AND DISCUSSION}

\section{A. Linking characteristic and simulated variables}

Now we proceed to demonstrate the link between characteristic variables $\left(Q_{c}, T_{f}^{90 \%}\right)$ and simulated variables $\left(\bar{Q}_{f}\right.$, $\left.T^{90 \%}\right)$. We aggregate the simulated exit node flow $Q$ observed through absolute simulation time $T$. Then, we level the basis for comparison between CAs by normalizing flow as $Q / Q_{c}$ and time as $T / T_{f}^{90 \%}$. We substitute $Q$ and $T$ for $\bar{Q}_{f}$ and $T^{90 \%}$

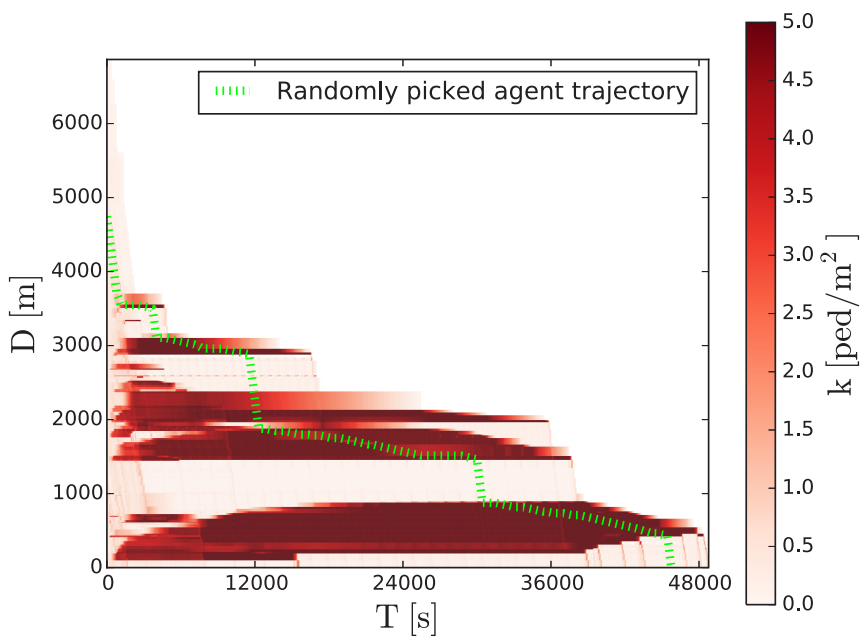

FIG. 5. Density $k$ at distance $D$ away from an exit node at time $T$ where the density ranges between $0.0 \leqslant k \leqslant 5.0 \mathrm{ped} / \mathrm{m}^{2}$ for a City of Bristol CA, specifically CA02 from Fig. 2. Trajectory of a randomly picked agent is shown in the green dotted path to illustrate how the agent velocity is reduced where the link density is high.

and define $\bar{Q}_{f}$ in relation to $Q_{c}$. We also define ratio $\bar{Q}_{f} / Q_{c}$ in relation to $T^{90 \%} / T_{f}^{90 \%}$. Using these relationships, we derive a general description of $T^{90 \%}$ using characteristic variables $Q_{c}$ and $T_{f}^{90 \%}$.

Aggregating simulated flow at exit node $Q$ across all CAs over absolute simulation time $T$ produces Fig. 6(a). Looking at the $0<T<20000$ band, we observe that the aggregate flows peak around $Q \approx 0.15 \mathrm{ped} /(\mathrm{ms})$ within a wide $68 \%$ confidence interval early on in the simulation which gradually tapers. While the peak signals the transition from the free flow $\left(T \leqslant T_{f}^{90 \%}\right)$ to the congested $\left(T>T_{f}^{90 \%}\right)$ regime, the exact point of transition is not clear in this representation. We also observe that as the sample size decreases with elapsing $T$, there is an increase in the fluctuation of aggregate $Q$. We normalize $T$ by $T_{f}^{90 \%}$ and $Q$ by $Q_{c}$ to obtain Fig. 6(b). $Q / Q_{c}$ clearly peaks within $T / T_{f}^{90 \%}<1$ at $Q / Q_{c} \approx 0.6$ which implies that, in general, $Q_{c}$ overpredicts the simulated flow. The flattening of the curve beyond the peak at $T / T_{f}^{90 \%} \geqslant 1$ indicates the congested flow regime which carries on up to a maximum of $T / T_{f}^{90 \%} \approx 72$. This is a significant gap between free-flow and simulated time but only applies to a small number of CAs.

For the following part, we randomly divide our 704 CAs into two halves of 352 , the first half to "train" our model and the second half to "test" it.

Using the train data set, we look at the extent to which $Q_{c}$ overpredicts $Q_{f}$ in Fig. 7(a). The upper bound appears to be defined by $\bar{Q}_{f}=Q_{c}$, showing that $\bar{Q}_{f}$ never exceeds $Q_{c}$. There is a strong agreement between $Q_{c}$ and $\bar{Q}_{f}$ along the diagonal where $Q_{c}<Q_{\max }$. However, when $Q_{c}>Q_{\max }$, $\bar{Q}_{f}$ diverges from the $\bar{Q}_{f}=Q_{c}$ line. It is better defined by a power-law fit $\left(r^{2}=0.79\right)$ described by Eq. (4) where $\theta=0.82$ and $\gamma=1.03$ :

$$
\bar{Q}_{f}=\gamma\left(Q_{c}\right)^{\theta}
$$



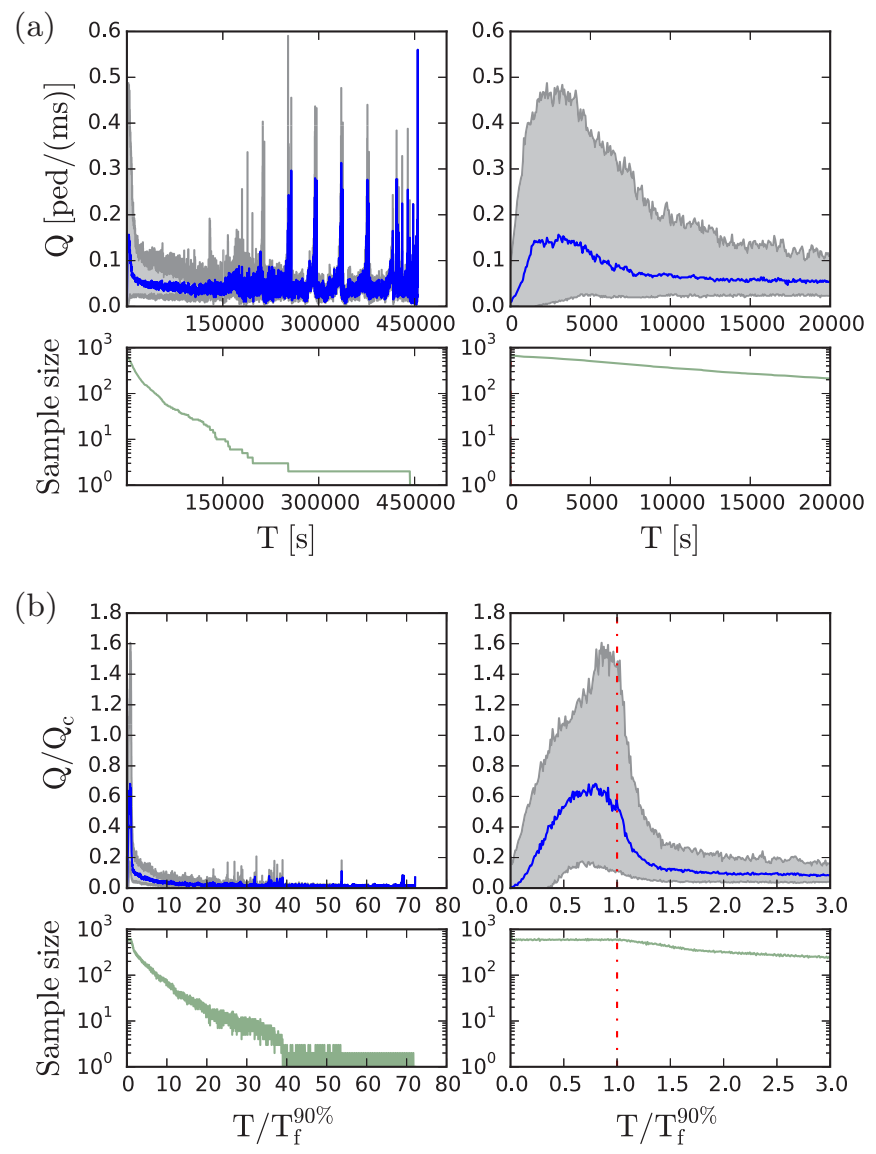

FIG. 6. (a) Aggregate simulated flow at exit node $Q$ averaged across all CAs over absolute simulation time $T$ as represented by the blue curve where the gray patch signifies the $68 \%$ confidence interval which becomes narrower with decreasing amount of aggregate data sample. (b) Aggregate exit node flow rate normalized by the characteristic flow rate, $Q / Q_{c}$, over time normalized by the free-flow time for $90 \%$ of all CA agents, $T / T_{f}^{90 \%}$, averaged across all CAs as represented by the blue curve showing the $68 \%$ confidence interval using the gray patch. $Q / Q_{c}$ peaks within $T / T_{f}^{90 \%}<1$ and mean $Q / Q_{c}<1$.

We look for an equation to estimate the ETE, i.e., $T^{90 \%}$, by analyzing the relationship between ratios $\bar{Q}_{f} / Q_{c}$ and $T^{90 \%} / T_{f}^{90 \%}$ representing $Q$ and $T$ as $\bar{Q}_{f}$ and $T^{90 \%}$, respectively. $\bar{Q}_{f} / Q_{c}$ estimates the peak of the mean curve in Fig. 6(b). For $T^{90 \%} / T_{f}^{90 \%} \gg 1$, delays due to agent interaction are proportionately greater and as such, $T^{90 \%} / T_{f}^{90 \%}=1$ is the best possible desired outcome.

We use the train data set to derive the relationship seen in Fig. 7(b) between $\bar{Q}_{f} / Q_{c}$ and $T^{90 \%} / T_{f}^{90 \%}$ with axes. There is a strong correlation $\left(r^{2}=0.73\right)$ between the data points. For values of $\bar{Q}_{f} / Q_{c} \approx 1, T^{90 \%} / T_{f}^{90 \%} \approx 1$ implying that $\bar{Q}_{f} \approx Q_{c}$ when $T^{90 \%} \approx T_{f}^{90 \%}$. However, $\bar{Q}_{f} / Q_{c} \rightarrow 0$ as $T^{90 \%} / T_{f}^{90 \%} \rightarrow$ $\infty$ since agents overflow into the congested regime. When $\bar{Q}_{f} \ll Q_{c}, T^{90 \%} \gg T_{f}^{90 \%}$. The relationship between the two ratios is well described by the power law of Eq. (5) with best
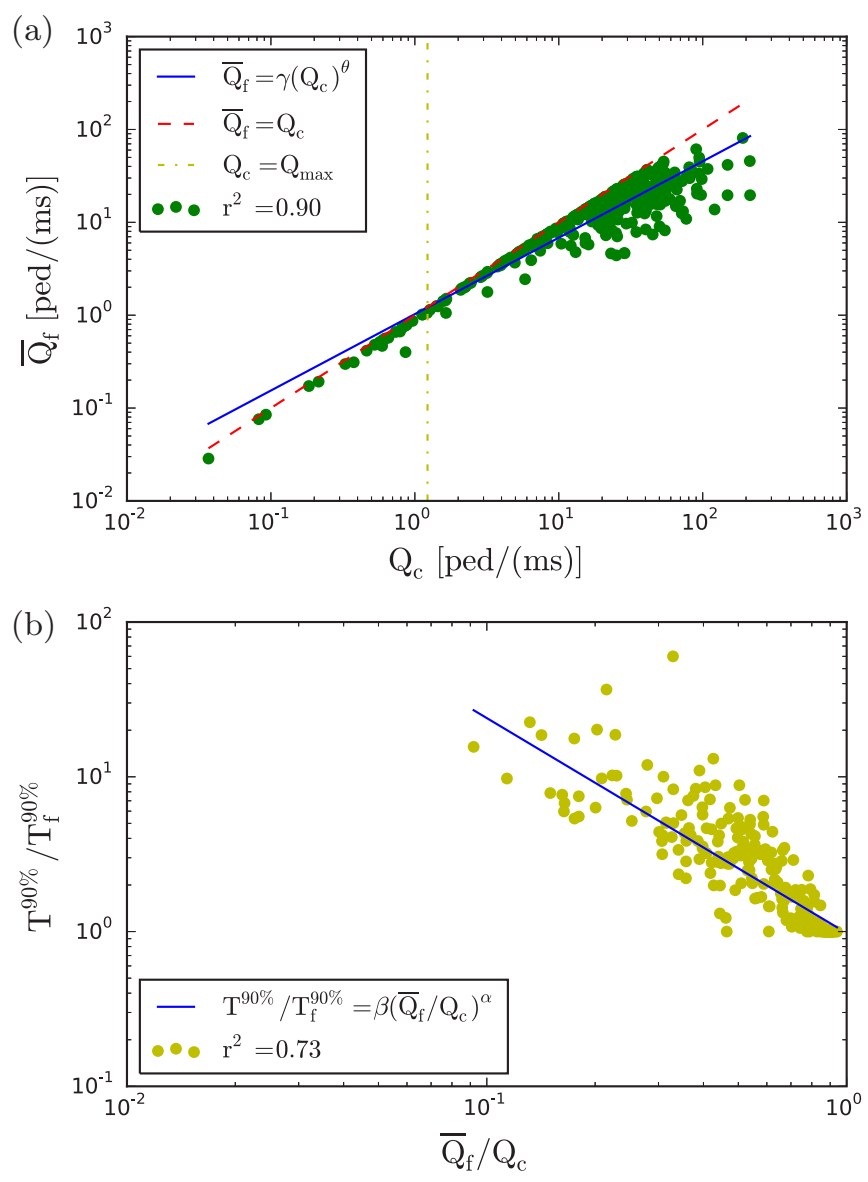

FIG. 7. (a) Relationship between characteristic flow $Q_{c}$ and mean of simulated exit node flow rate in the free-flow regime, $\bar{Q}_{f}$. Each CA is represented by a data point. $\bar{Q}_{f}=Q_{c}$ is the upper bound in the red dashed line. $\bar{Q}_{f}=\gamma\left(Q_{c}\right)^{\theta}$ describes the power-law best fit represented by the blue solid line. (b) Relationship between the ratio of mean simulated exit node flow rate in the free-flow regime to characteristic flow rate, $\bar{Q}_{f} / Q_{c}$, to the ratio of simulated time to free-flow time for $90 \%$ of all CA agents, $T^{90 \%} / T_{f}^{90 \%}$. The best fit shown by the blue solid line is a power-law equation $T^{90 \%} / T_{f}^{90 \%}=$ $\beta\left(\bar{Q}_{f} / Q_{c}\right)^{\alpha}$.

fit parameter values $\alpha=-1.39$ and $\beta=0.98$ :

$$
\frac{T^{90 \%}}{T_{f}^{90 \%}}=\beta\left(\frac{\bar{Q}_{f}}{Q_{c}}\right)^{\alpha} .
$$

In order to obtain at least the first order estimate of the ETE for a new CA without running an ABM simulation, we can equate $T^{90 \%}$ solely in terms of characteristic variables $T_{f}^{90 \%}$ and $Q_{c}$. We do this by substituting Eq. (4) into Eq. (5) to obtain Eq. (6) where $\phi=\alpha(\theta-1)=0.25$ and $\omega=\beta \gamma^{\alpha}=0.94$ :

$$
T^{90 \%}=\omega\left(Q_{c}\right)^{\phi} T_{f}^{90 \%}
$$

Substituting Eq. (3) into Eq. (6), we get Eq. (7):

$$
T^{90 \%}=\omega\left(\frac{N}{W}\right)^{\phi}\left(\frac{D^{90 \%}}{v_{\mathrm{f}}}\right)^{1-\phi}
$$




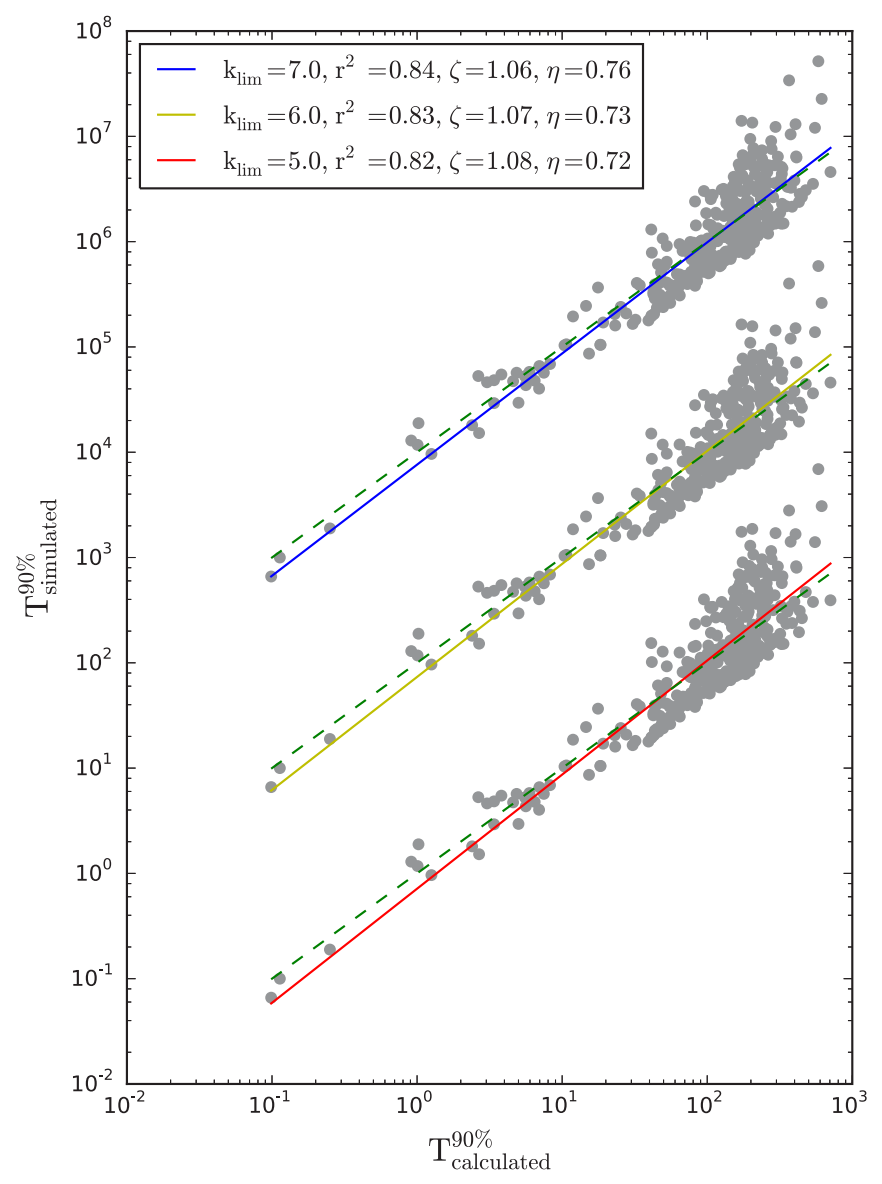

FIG. 8. Comparing $T_{\text {calculated }}^{90 \%}$ against $T_{\text {simulated }}^{90 \%}$ where $T_{\text {simulated }}^{90 \%}=$ $\eta\left(T_{\text {calculated }}^{90 \%}\right)^{\zeta}$. The red line represents the primary case where $k_{\text {lim }}=$ $5.0 \mathrm{ped} / \mathrm{m}^{2}$. The yellow and blue lines ( $y$-axis values offset by $10^{2}$ and $10^{4}$ ) represent secondary cases used for sensitivity analysis where $k_{\lim }=6.0$ and $7.0 \mathrm{ped} / \mathrm{m}^{2}$, respectively. The green lines are mirror diagonals where $T_{\text {simulated }}^{90 \%}=T_{\text {calculated }}^{90 \%}$ to allow visual comparison. There is a good fit between the axes as indicated by the strong $r^{2}$ values but higher values of $T_{\text {calculated }}^{90 \%}$ slightly underestimate $T_{\text {simulated }}^{90 \%}$.

\section{B. Verification and sensitivity analysis}

Using the test data set, we compare the simulated $T^{90 \%}$ values, $T_{\text {simulated }}^{90 \%}$, for each CA against their calculated counterpart,
$T_{\text {calculated }}^{90 \%}$, computed with the help of Eq. (7) using variables $N, W$, and $D^{90 \%}$ for each CA and constants $\omega, \phi$, and $v_{f}$. The relationship between $T_{\text {calculated }}^{90 \%}$ and $T_{\text {simulated }}^{90 \%}$ can be written as Eq. (8):

$$
T_{\text {simulated }}^{90 \%}=\eta\left(T_{\text {calculated }}^{90 \%}\right)^{\zeta}
$$

For the primary case where $k_{\lim }=5.0 \mathrm{ped} / \mathrm{m}^{2}$, there is a good agreement between $T_{\text {calculated }}^{90 \%}$ and $T_{\text {simulated }}^{90 \%}\left(r^{2}=0.82\right)$ as demonstrated by Fig. 8 . Since the gradient of the best fit line crosses the mirror diagonal line when $T_{\text {calculated }}^{90 \%}=e^{\frac{\ln \zeta}{1-\eta}}$, we can show that $T_{\text {calculated }}^{90 \%}$ slightly underestimates $T_{\text {simulated }}^{90 \%}$ (exponent $\zeta=1.08$, coefficient $\eta=0.72$ ) for values of $T_{\text {calculated }}^{90 \%}>$ $10^{1.78}$

Our results do not appear to be sensitive to $k_{\text {lim }}$ since raising the density limit from 5.0 to 6.0 then to $7.0 \mathrm{ped} / \mathrm{m}^{2}$ only changes the exponent $\zeta$ by $1 \%$ in both of our secondary cases (from 1.08 to 1.07 then to 1.06) as visible in Fig. 8. So, our results appear to be robust within these density limit conditions marked in Fig. 1.

\section{CONCLUSIONS}

In conclusion, by exploring the relationship between simulated ETE and CA attributes from 50 UK cities, we present a way to estimate ETEs using CA attributes alone: population, CA size, and exit width. This method is even more reliable for CAs with a characteristic flow rate lower than the maximum flow rate of $1.22 \mathrm{ped} / \mathrm{ms}$, and the results appear to be robust within the range of density limit conditions explored. We envisage our method being used to rapidly evaluate lots of evacuation strategies and the best candidates among these examined using ABM simulations to determine a more specific answer. In our future work, we want to establish dominant topological attributes that are able to account for the unique conditions of each CA so that we can correct for discrepancies which statistical analyses are not always able to capture.

\section{ACKNOWLEDGMENTS}

B.K. is grateful for funding from an EPSRC Doctoral Training Grant No. EP/K502996/1 and a University of Bristol Systems Centre Open Innovation Industry Scholarship.
[1] Department of Economic and Social Affairs, United Nations, World economic and social survey 2013: Sustainable development challenges (United Nations, New York, 2013).

[2] Munich RE, Topics geo: Natural catastrophes 2013: Analyses, assessments, positions, edited by W. Kron and A. Schuck (Munchener Ruckversicherungs-Gesellschaft, München, 2014).

[3] T. Urbanik II, J. Hazard. Mater. 75, 165 (2000).

[4] B. Kolen and I. Helsloot, Disasters 36, 700 (2012).

[5] M. K. Lindell, Transp. Res. Part A: Policy Pract. 42, 140 (2008).

[6] Z. Sinuany-Stern and E. Stern, Socio-Econ. Plann. Sci. 27, 97 (1993).

[7] Y. Sheffi, H. Mahmassani, and W. B. Powell, Transp. Res. Part A: Gen. 16, 209 (1982).
[8] T. Urbanik II and A. E. Desrosiers, An Analysis of Evacuation Time Estimates Around 52 Nuclear Power Plant Sites Analysis and Evaluation, Technical Report (Pacific Northwest National Laboratory, Richland, WA, USA, 1981), http://pbadupws.nrc.gov/docs/ML1325/ML13259A182.pdf.

[9] S. Bretschneider and A. Kimms, Transp. Res. Part A: Policy Pract. 45, 523 (2011).

[10] Y.-C. Chiu, H. Zheng, J. Villalobos, and B. Gautam, IIE Trans. 39, 83 (2007).

[11] M. D’Orazio, E. Quagliarini, G. Bernardini, and L. Spalazzi, Int. J. Disaster Risk Reduct. 10, Part A, 153 (2014).

[12] G. Lämmel, D. Grether, and K. Nagel, Transp. Res. Part C: Emerging Technol. 18, 84 (2010). 
[13] P. M. Simon, J. Esser, and K. Nagel, Int. J. Mod. Phys. C 10, 941 (1999).

[14] T. Yamada, Int. J. Syst. Sci. 27, 931 (1996).

[15] J. K. Riad, F. H. Norris, and R. B. Ruback, J. Appl. Soc. Psychol. 29, 918 (1999).

[16] E. J. Baker, Mass Emerg. 4, 9 (1979).

[17] A. J. Pel, S. P. Hoogendoorn, and M. C. Bliemer, Procedia Eng. 3, 101 (2010).

[18] A. Johansson, M. Batty, K. Hayashi, O. Al Bar, D. Marcozzi, and Z. A. Memish, Lancet Infect. Dis. 12, 150 (2012).

[19] M. Bukáček, P. Hrabák, and M. Krbálek, Transp. Res. Procedia 2, 105 (2014).

[20] A. Johansson, Phys. Rev. E 80, 026120 (2009).

[21] D. Helbing, I. J. Farkas, P. Molnar, and T. Vicsek, Pedestrian and Evacuation Dyn. 21, 21 (2002).

[22] D. Helbing, A. Johansson, J. Mathiesen, M. H. Jensen, and A. Hansen, Phys. Rev. Lett. 97, 168001 (2006).

[23] D. Helbing, A. Johansson, and H. Z. Al-Abideen, Phys. Rev. E 75, 046109 (2007).

[24] D. Helbing, I. Farkas, and T. Vicsek, Nature (London) 407, 487 (2000).
[25] U. Weidmann, Transporttechnik der Fussgänger: Transporttechnische Eigenschaften des Fussg ängerverkehrs (Literaturauswertung) (ETH, IVT, Zurich, 1993).

[26] B. Kunwar, F. Simini, and A. Johansson, Transp. Res. Procedia 2, 813 (2014).

[27] E. W. Dijkstra, Numer. Math. 1, 269 (1959).

[28] M. Haklay and P. Weber, IEEE Pervasive Comput. 7, 12 (2008).

[29] M. F. Goodchild, GeoJournal 69, 211 (2007).

[30] M. Haklay, Environ. Plann. B, Plann. Des. 37, 682 (2010).

[31] P. Neis and A. Zipf, ISPRS Int. J. Geo-Inf. 1, 146 (2012).

[32] A. L. Ali, F. Schmid, R. Al-Salman, and T. Kauppinen, in Proceedings of the 22nd ACM SIGSPATIAL international conference on advances in geographic information systems, SIGSPATIAL '14 (ACM, New York, NY, USA, 2014), pp. 143-152.

[33] D. L. Balk, U. Deichmann, G. Yetman, F. Pozzi, S. I. Hay, and A. Nelson, Advances in Parasitology 62, 119 (2006).

[34] Office for National Statistics, National Population Projections, 2010-Based Statistical Bulletin, Technical Report (Office for National Statistics, Newport, United Kingdom, 2011). 